\title{
Breast Cancer Diagnosis Using Microwave AND HYBRID IMAGING METHODS
}

\author{
Younis M. Abbosh \\ College of Electronics Engineering, Mosul University, Mosul, Iraq
}

\begin{abstract}
This paper explains new imaging techniques that show promising results in breast cancer detection. The presented techniques use microwave-based methods, wavelet analyses, and neural networks to get a suitable resolution for the breast image. One of the presented techniques (hybrid method) uses a combination of microwaves and acoustic signals to improve the detection capability. Some promising results are shown and explained.
\end{abstract}

\section{KEYWORDS}

microwav imaging; breast cancer; hybrid imaging ; neural network; wavelet.

\section{INTRODUCTION}

Breast cancer is one of the most common cancers diagnosed in women. In the western countries, it is estimated that one in eleven women will develop breast cancer at some stage in their life making it the highest life risk [1,2]. Early detection and timely medical treatment are key factors affecting long-term survival of breast-cancer patients. Currently, the primary method for breast screening is X-ray mammography and in some cases magnetic resonance imaging (MRI). $\mathrm{X}$-ray mammography is the golden diagnostic tool for breast cancer, but the technology has several serious shortfalls. Firstly, it produces a relatively high false-negative rate, which can be as large as $30 \%$ [3]. Secondly, screening mammography suffers from a high false-positive rate: on average, $75 \%$ of breast biopsies prompted by a "suspicious" mammographic abnormality prove benign [4]. Thirdly, screening mammography is less sensitive in women with radio-graphically dense breast tissue prevalent in younger women, where it has been shown that X-ray mammography has failed to detect up to $30 \%$ of cancers greater than $5 \mathrm{~mm}$ in diameter [5], due to its relatively poor soft-tissue contrast.

Mammography's other drawbacks include variability in radiological interpretation, and a slight risk of inducing cancer due to the ionizing radiation exposure. Frequent monitoring is difficult because of health concerns related to exposure to ionizing radiation [6]. Best prospects of detecting malignant tissues using X-rays are when tumours reach the late stage of calcification, which seriously jeopardises the success of treatment.

MRI is a highly sensitive imaging technique, but lacks information on specificity, and thus it has high rate of false positive results [7]. The impact of misdiagnosis on the patient is considerable, particularly when they are confronted with unnecessary surgeries. In general, MRI can be considered to have a moderate success rate of correct diagnosis; however MRI has high operational costs. 
International Journal of Computer Science \& Engineering Survey (IJCSES) Vol.5, No.3, June 2014

An ultimate diagnosis of all types of breast disease depends on a biopsy. A biopsy is an invasive procedure to remove and examine tissue or cells for the presence of cancer. In most cases the decision for a biopsy is based on mammography findings. Unfortunately, biopsy results indicate that $80 \%$ of breast lesions detected by mammography are benign. This situation calls for alternative diagnostic tools to reduce physical and mental suffering of patients caused by this false positive diagnosis. Of particular interest is the development of low-cost diagnosis methods, which could be easily accessed by as much people as possible.

Given the deficits of the current diagnostic tools, development of imaging modalities that enhance, complement, or replace X-ray mammography or MRI has been a priority in many countries and by many research groups around the globe. Some of the alternative modalities under investigation by research groups around the world are ultrasound-based and microwavebased methods.

Ultrasound imaging of the breast is capable of distinguishing between solid tumours and fluid-filled cysts. Also, it can be used to evaluate lumps that are hard to see on a mammogram. As ultrasound does not harm biological tissue, thus it can be applied frequently. This is of importance, especially with respect to younger women for whom the risks from X-ray radiation are most significant. However, ultrasound lacks spatial resolution, cannot image calcifications and is very operator dependent. Breast cancer is relatively iso-echoic [8] (that is, the echo similarity of two or more tissues as measured by ultrasonography) and hence, difficult to detect by ultrasound. Thus, unnecessary biopsies are needed on benign masses [8].

In the last years, intensive research has been conducted at many universities on different microwave-based imaging techniques for breast cancer detection. Some of promising techniques use suitable parameters extracted from microwave signal collected at different places around breast as input to neural network. These parameters are extracted from time and frequency domain using wavelet transforms [9-11]. The breast model including the probes that are used to collect the scattered signals is analyzed using the full-wave electromagnetic simulator CST Microwave Studio. This research has enjoyed notable success especially in the design, and development of different devices used in those techniques [12-20]. It has been demonstrated that the use of microwave technology, combined with acoustic signals, properly designed microwave devices can improve the resolution needed for early breast cancer detection. Those systems are still under continuous improvements aiming at reaching a standard required to make the system clinically reliable.

\section{MicROWAVE IMAgING}

The potential for using microwaves for detecting breast tumours is based on the concept of tissue-dependent microwave scattering and absorption in the breast to exploit the contrast in the dielectric properties of malignant and normal breast tissues. There are many approaches in which microwaves can be utilized in the imaging tools as shown in Fig. 1 [30].

It has been widely assumed that normal breast tissue is largely transparent to microwaves because they are featured with a low relative permittivity and conductivity at the microwave frequency bands, whereas lesions, which contain more water and blood are characterized by a high relative permittivity and conductivity at the microwave frequencies and hence they cause a significant backscatter [21]. Upon this assumption, which is supported by some measurements, microwave imaging systems are being designed to detect the presence of a small object inside a breast causing a considerably larger backscatter than the surrounding medium. 
International Journal of Computer Science \& Engineering Survey (IJCSES) Vol.5, No.3, June 2014

Microwave techniques involve the propagation of very low levels (1000 times less than a mobile phone) of microwave energy through the breast tissue. The basis for tumour detection and location is the difference in the electrical properties between normal and malignant breast tissue. Normal breast tissue is largely transparent to microwave radiation, whereas malignant tissues, which contain more water and blood, cause microwave signal backscattering. This scattered signal can be picked by an array of microwave antennas and analysed using a computer.

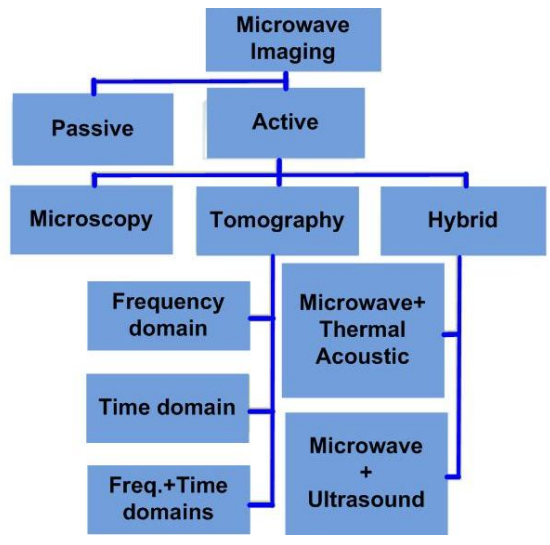

Figure. 1 Microwave-based imaging methods

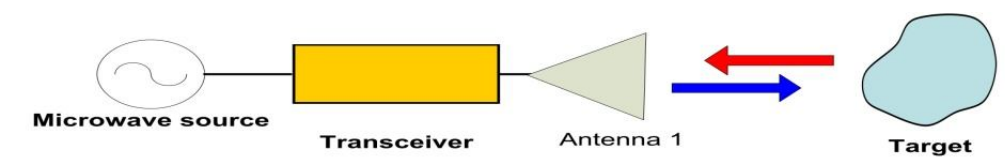

(a) Monostatic

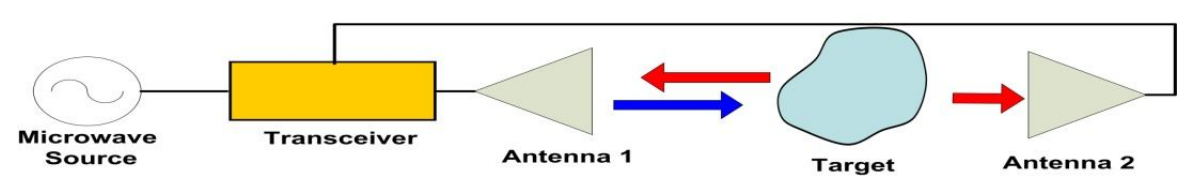

(b) Bistatic

Figure. 2 Radar-based microwave imaging system of (a) monostatic, and (b) bistatic radars.

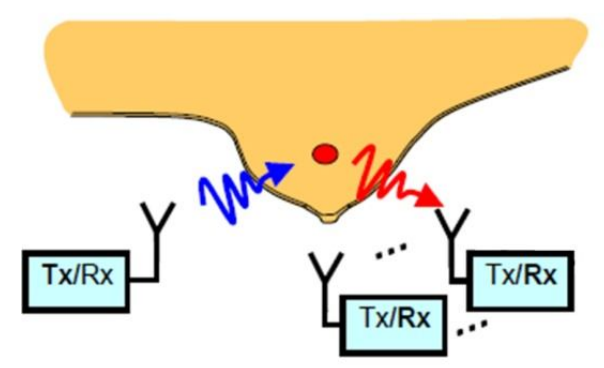

Figure. 3 Radar-based imaging of breast. 


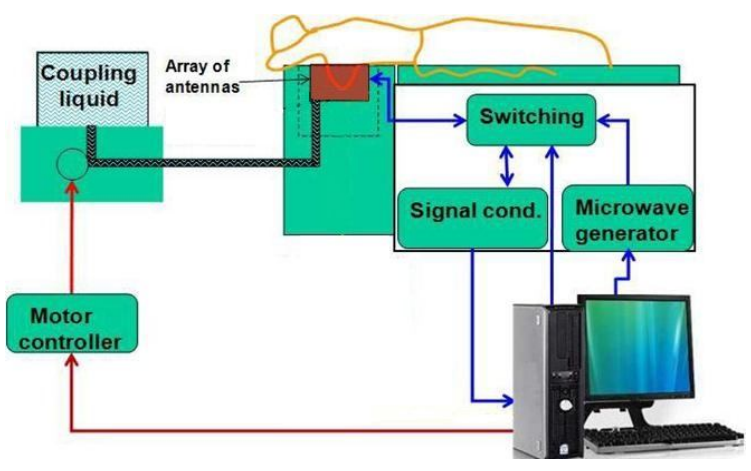

Figure. 4 Configuration of the radar system.

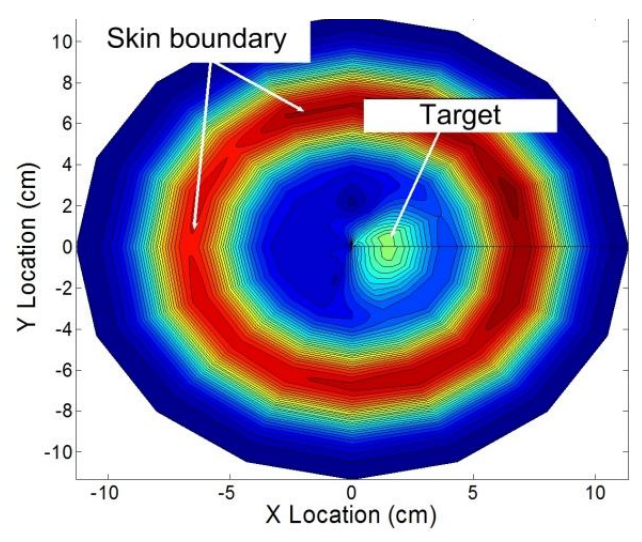

Figure. 5 Imaging result of the wideband radar system.

The radar approach to microwave imaging (Fig. 2) employs generating and receiving short pulses for various locations of probe antenna or alternatively by an array antenna. The processed signals for various locations of a probe antenna or from array elements are combined to form a two or three-dimensional image showing the location of a highly reflecting object representing a cancerous tissue [12].

The configuration, shown in Fig. 2(a), is based on the principle of monostatic radar. In this configuration, the same antenna is used for both transmitting and receiving of a microwave signal. The configuration shown in Fig. 2(b) uses two antennas, which are displaced by a certain distance. In this case, the microwave imaging system is based on the principle of bistatic radar.

When using the radar-based techniques to image human breast, the procedure is as shown in Fig. 3 [30]. The configuration of a prototype radar system is shown in the Fig. 4 [16]. The system consists of a circular cylindrical scanning platform with a resolution of $1^{\circ}$ to support a breast phantom, and a mechanical scanning platform with resolution of $0.1 \mathrm{~mm}$ in the vertical axis. The scanning platform supports an array of wideband antennas [22]. The antenna is connected to a microwave Vector Network Analyzer that measures the scattered signals. The collected scattered signals are then processed in a personal computer to get an image of the breast.

The imaging capabilities of the aforementioned radar system are carried out for an artificial breast phantom. Sample of the obtained images is shown in Fig. 5. The figure clearly shows the boundaries of the layer representing the skin, size and shape of the object that represents the tumor. 
International Journal of Computer Science \& Engineering Survey (IJCSES) Vol.5, No.3, June 2014

The microwave-based imaging systems face two serious challenges before being ready for clinical tests. First, the success of those systems depends on a substantial contrast in the dielectric properties of malignant and normal breast tissues. A large scale study to experimentally determine the dielectric properties of a variety of normal, malignant and benign breast tissues, measured at the microwave range has recently been conducted in the USA [23, 24]. It has been demonstrated that the dielectric contrast varies widely from individual to individual and with age and some other biological factors. It has also been found that although the contrast between malignant and normal adipose-dominated tissues in the breast is considerable, the contrast in the dielectric properties is no more than about $10 \%$ between malignant and normal glandular/fibro-connective tissues in the breast [24]. By using the scattering theory of electromagnetic fields [25], it is possible to show that when the dielectric contrast between the normal and malignant tissue is low, resolution of the microwave imaging system deteriorates rapidly resulting in a blurry image of the breast.

The second important challenge that limits the success of the microwave-based imaging systems originates from the nature of the microwave signals. The limited resolution comes from the fact that the heterogeneous structure of the breast causes multiple scatterings and reflections for the microwave signal while penetrating inside the tissues. This increases the uncertainty in estimating the three-dimensional image of the breast. That uncertainty can only be removed by additional information about the breast tissues.

\section{HYBRID IMAGING}

In order to remove the obstacles facing the success of the microwave-based imaging methods, the hybrid technique is proposed and tested $[17,19,20]$. The hybrid technique utilizes the dielectric (electrical properties) and elasticity (mechanical properties) contrast between tumors and healthy tissue in order to produce a three dimensional image of the breast. The information from the hybrid image significantly enhances early diagnostic accuracy.

The mechanical properties of biological tissues are important indicators for biomedical diagnosis since they are generally correlated with tissue pathological changes [26]. For example, different kinds of carcinomas of the breast have been found to be harder than its surrounding normal tissues [27]. Although different in terms of their elasticity, some tumors are not readily detectable by conventional imaging modalities, especially in the presence of complex background alterations such as scar tissue, or other benign phenomena [28].

A schematic diagram of the system is shown in Fig. 6 [17-20]. Under operator guided computer control, a very short frequency-modulated pulse is applied from an ultra wideband (UWB) antenna beside the breast of a patient in the face-down (prone) position via a switching unit. At the same time, an acoustic signal is applied from a transducer below the breast. The proposed hybrid system utilizes the combined benefits of microwaves, with UWB spectrum, and acoustic excitations to produce a three-dimensional image. In this method, microwaves are employed to give a full view of the dielectric contrasts, while acoustics give the elasticity distributions within the breast. The two distributions are then used to produce a final image with high contrast and resolution.

The scattered signals, which are a consequence of the difference in the electrical and mechanical properties of different types of breast tissues, are collected by a cylindrical array of UWB antennas encircling the breast. Parameters of the scattered signals, i.e. amplitude, time delay, frequency shift and their angular distribution, correlate with characteristics of the breast tissue. For example, shift in frequency, which is called Doppler frequency, is due to elasticity contrast, whereas time delay is due to dielectric contrast. The detection unit shown in Fig. 6 
captures those parameters, and the signal conditioning unit is designed to digitize and improve the quality of the data for storage on a high speed computer. Operation of the different modules of the system are controlled from a computer, which would also be used for calibration, data collection, processing, and to display the images. Certain processing algorithms are used to produce a clear three-dimensional image of the breast. The operator is able to distinguish tumors from the healthy tissues of the breast by identifying the different breast regions characterized by high backscatter and high stiffness.

The switching unit depicted in Fig. 6 includes a matrix of directional couplers and power dividers. The unit is used to direct the generated microwave pulse towards the transmitting antenna and the scattered pulses towards the detection unit and achieve a high level of isolation between them. The detection unit includes a matrix of correlators, which have the ability to detect the amplitude and phase variations of the received signals.

In order to remove undesired reflections due to the mismatch between the antenna, the skin layer and the space separating them, the breast under test is immersed in a coupling liquid which has material properties that reduce the backward scattering at the skin layer, and thus increasing the dynamic range of the system [29].

Snapshots from the results of imaging using the hybrid technique are shown in Fig. 7. The results in that figure reveal the possibility of detection as small as $1 \mathrm{~mm}$ tumors. This is a promising result. However, further work needs to be done so that the imaging can be performed in more realistic environments using heterogeneous breast phantoms.

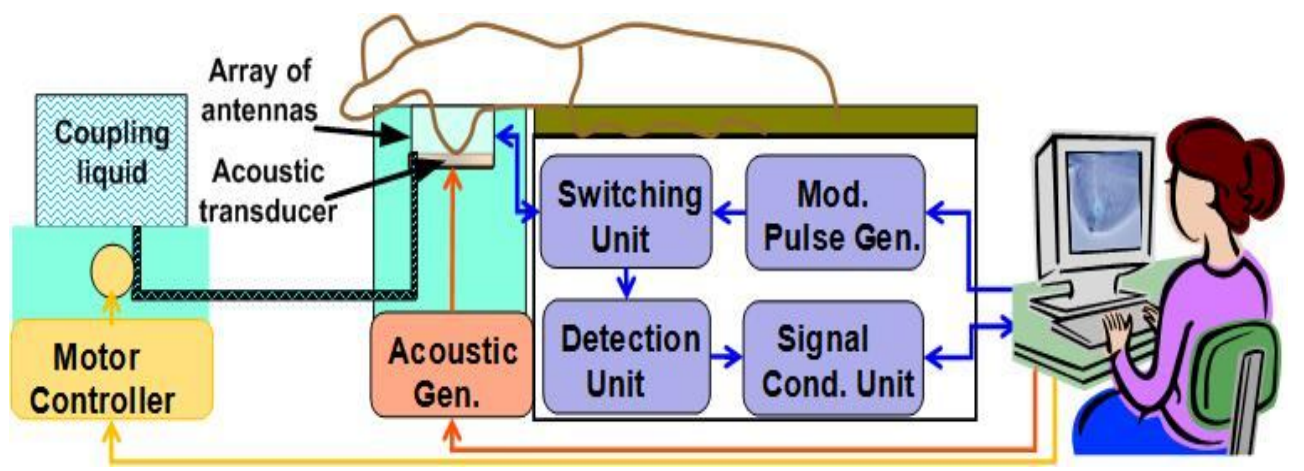

Figure. 6 A schematic diagram showing the hybrid imaging system [14], [16], [17]. 
International Journal of Computer Science \& Engineering Survey (IJCSES) Vol.5, No.3, June 2014
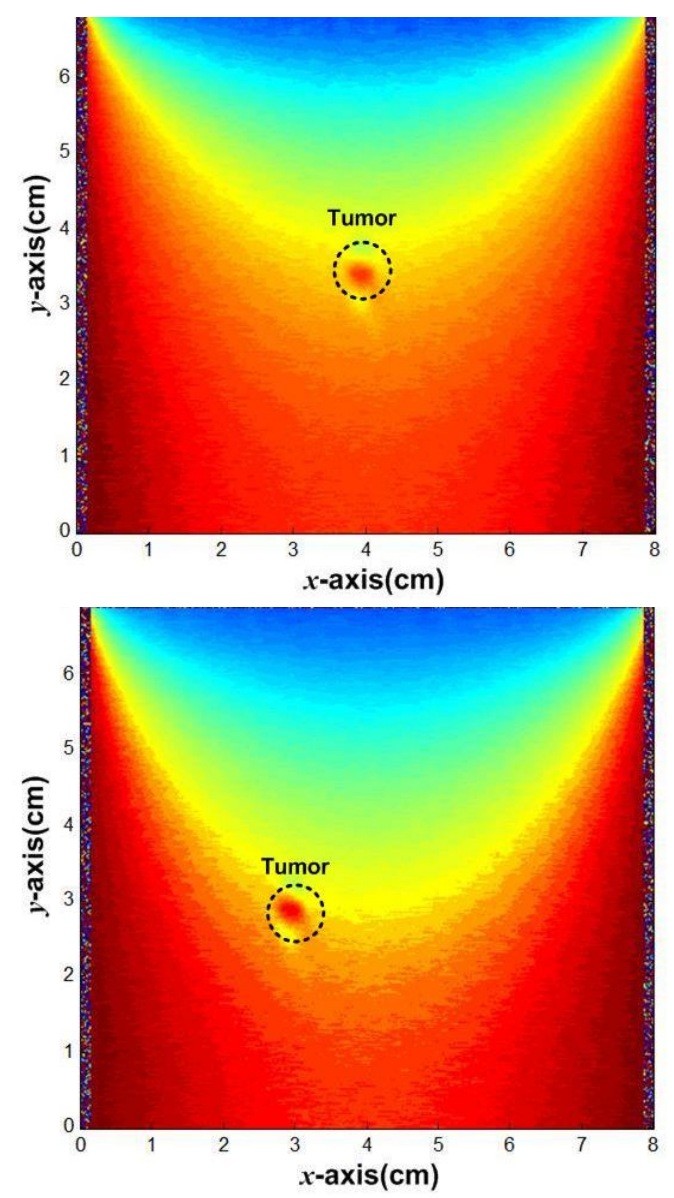

Figure. 7 The successful detection of small tumors using the hybrid techniques on artificial breast phantom.

\section{REFERENCES}

[1] Australian Institute of Health and Welfare \& Australasian Association of Cancer Registries (AACR), "Cancer in Australia 2001" AIHW Cat. \# CAN23, Canberra: AIHW (Cancer Series no. 28), pp64-68, 2004

[2] Breast cancer network Australia, http://www.bcna.org.au/content/view/526/588/, 2008.

[3] [23] P. Huynh, A. Jarolimek, and S. Daye, “The false-negative mammogram," Radiographics, vol. 18, no. 5, pp. 1137-1154, 1998.

[4] S. Nass, I. Henderson, and J. Lashof, Mammography and beyond: Developing technologies for the early detection of breast cancer, National Academy Press, Washington D.C., 2001.

[5] E. A. Sickles, "Nonpalpable, circumscribed, noncalcified, solid breast masses: Likelihood of malignancy based on lesion size and age of patient," Radiology, vol. 192, pp. 439-442, 1994.

[6] E. Fear, P. Meaney, and M. Stuchly, "Microwaves for breast cancer detection" IEEE Potentials, vol. 22, no. 1, pp. 12-18, 2003.

[7] N. Hoogerbrugge, et al, "The impact of a false-positive MRI on the choice for mastectomy in BRCA mutation carriers is limited," Annals of Oncology, vol. 19, no. 4, pp. 655-659, 2008.

[8] D. Melodelima, J. Bamber, F. Duck, J. Shipley, and L. Xu, "Elastography for breast cancer diagnosis using radiation force: system development and performance evaluation," Ultrasound in Medicine and Biology, vol. 32, no. 3, pp. 387-396, 2006.

[9] Younis M. Abbosh, Ammar F. Yahya, , and Amin Abbosh, "Neural Networks for the Detection and Localization of Breast Cancer," International Conference on Communications and Information Technology (ICCIT), pp 156-159, March 2011 
International Journal of Computer Science \& Engineering Survey (IJCSES) Vol.5, No.3, June 2014

[10] Ammar F. Yahya, Younis M. Abbosh, and Amin Abbosh, "Microwave Imaging Method Employing Wavelet Transform and Neural Networks for Breast Cancer Detection," Proceedings of the AsiaPacific Microwave Conference 2011, pp 1418-1421

[11] A. Abbosh, Y. Abbosh, and A. Yahya "Modeling and Imaging of Breast Using the Combined Effect of Microwave and Acoustic Pulses," Proceedings of the Asia-Pacific Microwave Conference 2011, pp 1790-1793

[12] W. Khor, M. Bialkowski, A. Abbosh, N. Seman, and S. Crozier, "An ultra wideband microwave imaging system for breast cancer detection," IEICE Trans. Communications, vol. E-90B, no. 9, pp. 2376-2381, 2007.

[13] A. Abbosh, K. Kan, and M. Bialkowski, "A compact UWB planar tapered slot antenna for use in a microwave imaging system," Microwave and Optical Technology Letters, vol. 48, no. 11, pp. 22122216, 2006.

[14] W. Khor, H. Wang, M. Bialkowski, A. Abbosh, and N. Seman, "An experimental and theoretical investigation into capabilities of a UWB microwave imaging radar system to detect breast cancer," European Microwave Conference, 2007.

[15] A. Abbosh, M. Bialkowski, and S. Crozier, "A simple model for electromagnetic scattering due to breast tumour," IEEE-Antennas and Propagation Symposium, 2008.

[16] W.C. Khor and M.E. Bialkowski, "Investigations into Cylindrical and Planar Configurations of a Microwave Imaging System for Breast Cancer Detection" Proc. IEEE Antennas and Propagation Society International Symposium, pp. 263-266, Albuquerque, New Mexico, USA, Jul 2006.

[17] A. Abbosh, S. Crozier, "Strain Imaging of the Breast by Compression Microwave Imaging," Antennas and Wireless Propagation Letters, IEEE, vol.9, no., pp.1229-1232, 2010

[18] A. Abbosh, and S. Crozier, "Hybrid Imaging Method for Early Breast Cancer Detection," Biomedical Engineering Conference, 2008. CIBEC 2008. Cairo International , vol., no., pp.1-4, 18-20 Dec. 2008

[19] A. Abbosh, "Strain imaging of breast using ultra-wideband pulse," Microwave Conference Proceedings (APMC), 2010 Asia-Pacific , vol.,

[20] A. Abbosh, "Early breast cancer detection using hybrid imaging modality," Antennas and Propagation Society International Symposium, 2009. APSURSI '09. IEEE, vol., no., pp.1-4, 1-5 June 2009

[21] Y. Xie, B. Guo, L. Xu, J. Li, and P. Stoica, "Multistatic adaptive microwave imaging for early breast cancer detection," IEEE Trans. Biomed. Eng., vol. 53, pp. 1647-57, 2006.

[22] A. Abbosh, H.K. Kan and M.E. Bialkowski "Design of compact directive ultra wideband antipodal antenna" Microwave and Optical Techn. Letters. vol. 48, No. 12, pp. 2448-2450, 2006.

[23] M. Lazebnik, et al, "A large-scale study of the ultrawideband microwave dielectric properties of normal breast tissue obtained from reduction surgeries," Physics in Medicine and Biology, vol. 52, pp. 2637-2656, 2007.

[24] M. Lazebnik, et al, "A large-scale study of the ultrawideband microwave dielectric properties of normal, benign and malignant breast tissues obtained from cancer surgeries," Physics in Medicine and Biology, vol. 52, pp. 6093-6115, 2007.

[25] D. Fotiadis, and C. Massalas, Mathematical Methods in Scattering Theory and Biomedical Engineering, World Scientific, 2007.

[26] Y. Fung, Biomechanics: Mechanical properties of living tissues, 2nd ed., Springer-Verlag, New York, 1993.

[27] W. Donegan, J. Spratt, and J. Orsini, Cancer of the breast, 5th ed., W.B. Saunders, New York, 1987.

[28] L. Newcomer, et al, 'Detection method and breast carcinoma histology,' Cancer, vol. 95, pp. 470477, 2002.

[29] A. Abbosh, M. Bialkowski, and S. Crozier, "Investigations into optimum characteristics for the coupling medium in UWB breast cancer imaging systems", IEEE-Antennas and Propagation Symposium, USA, 2008.

[30] V. Zhurbenko, "Challenges in the Design of Microwave Imaging Systems for Breast Cancer Detection”, Advances in Electrical and Computer Engineering, vol. 11, no. 1, pp. 91-96, 2011.

\section{Author}

Younis M. Abbosh born in 1957 in Mosul-IRAQ. He awarded BSc in Electronic and Communication Engineering from University of Mosul, Mosul- IRAQ in 1979.Next, he awarded the MSc in Electronic and Communication from University of Mosul in 1985, and $\mathrm{PhD}$ in Electronic and Communication in 2006 from same University. From 20072010, Dr Younis worked as assistance dean of College of Electronics Engineering at Mosul University. From 2013-now he is working as Head of Computer and Information Department in College of Electronics Engineering.

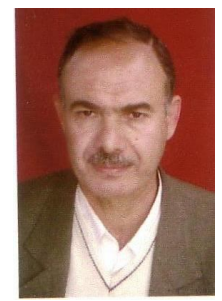

Through his academic life he published over 10 papers in field of Electronics, Communications, and Digital Signal Processing. 